University of Nebraska - Lincoln

DigitalCommons@University of Nebraska - Lincoln

Faculty Publications from Nebraska Center for

Materials and Nanoscience

Materials and Nanoscience, Nebraska Center

for (NCMN)

6-23-2005

\title{
Effective electromechanical moduli of ferroelectric ceramics with fiber textures
}

Y.C. Zhou

Xiangtan University, China

J. Liu

University of Colorado-Boulder

Jiangyu Li

University of Nebraska-Lincoln, jjli@uw.edu

Follow this and additional works at: https://digitalcommons.unl.edu/cmrafacpub

Part of the Nanoscience and Nanotechnology Commons

Zhou, Y.C.; Liu, J.; and Li, Jiangyu, "Effective electromechanical moduli of ferroelectric ceramics with fiber textures" (2005). Faculty Publications from Nebraska Center for Materials and Nanoscience. 42.

https://digitalcommons.unl.edu/cmrafacpub/42

This Article is brought to you for free and open access by the Materials and Nanoscience, Nebraska Center for (NCMN) at DigitalCommons@University of Nebraska - Lincoln. It has been accepted for inclusion in Faculty Publications from Nebraska Center for Materials and Nanoscience by an authorized administrator of DigitalCommons@University of Nebraska - Lincoln. 


\title{
Effective electromechanical moduli of ferroelectric ceramics with fiber textures
}

\author{
Y. C. Zhou \\ Key Laboratory of Advanced Materials \& Rheological Properties of Ministry of Education, \\ Faculty of Materials \& Optoelectronic Physics, Xiangtan University, China \\ J. Liu \\ Department of Mechanical Engineering, University of Colorado-Boulder \\ J. Y. Li ${ }^{a), b)}$ \\ Department of Engineering Mechanics and Center for Materials Research and Analysis, \\ University of Nebraska-Lincoln
}

(Received 24 January 2005; accepted 15 May 2005; published online 24 June 2005)

In this letter, we report the predictions on the effective piezoelectric coefficients and electromechanical coupling factors of ferroelectric ceramics $\mathrm{BaTiO}_{3}$ and $\mathrm{Pb}\left(\mathrm{Mg}_{1 / 3} \mathrm{Nb}_{2 / 3}\right) \mathrm{O}_{3}-\mathrm{PbTiO}_{3}$ (PMN-PT) with various fiber textures, including [001], [011], and [111], using a two-scale micromechanical model that accounts for microstructural phenomena at both domain and grain levels. It was observed that for $\mathrm{BaTiO}_{3}$ the [011] texture is optimal with highest $d_{31}$ and $d_{33}$, while for PMN-PT [001] texture is optimal despite the fact that [011]-oriented single-crystalline PMN-PT has higher $d_{32}$ than that of [001]-oriented PMN-PT single crystal. In fact, [011]-textured PMN-PT ceramics have much smaller piezoelectric coefficients $d_{32}$ and $d_{33}$ than does [011]-oriented PMN-PT single crystal. It is also noted that [001]-textured $\mathrm{BaTiO}_{3}$ and $\mathrm{PMN}-\mathrm{PT}$ ceramics have even higher electromechanical coupling factor $k_{31}$ than that of [001]-oriented single crystals. () 2005 American Institute of Physics. [DOI: 10.1063/1.1952584]

Ever since the discovery of ultrahigh strain and piezoelectric behavior in relaxor-based [001]-oriented rhombohedral single crystals $\mathrm{Pb}\left(\mathrm{Mg}_{1 / 3} \mathrm{Nb}_{2 / 3}\right) \mathrm{O}_{3}-\mathrm{PbTiO}_{3}$ (PMN-PT) and $\mathrm{Pb}\left(\mathrm{Zn}_{1 / 3} \mathrm{Nb}_{2 / 3}\right) \mathrm{O}_{3}-\mathrm{PbTiO}_{3}(\mathrm{PZN}-\mathrm{PT}),{ }^{1,2}$ there have been efforts devoted to accomplishing similar electromechanical properties in textured polycrystalline ceramics, ${ }^{3-5}$ which are relatively cheaper to produce. Texture levels up to $90 \%$ and piezoelectric coefficients as high as $90 \%$ of single-crystal values have been demonstrated in the resulting ceramics, ${ }^{6}$ which are very attractive for transducer applications. However, most of these investigations focused on [001]-textured PMN-PT ceramics, and other textures have rarely been studied. On the other hand, there is compelling experimental and theoretical evidence suggesting that single-crystalline PMN-PT and PZN-PT oriented along other directions, such as [011] axis, may have even higher electromechanical coupling. For example, much higher piezoelectric coefficient $d_{32}$ and electromechanical coupling factor $k_{32}$ were observed in [011]-oriented PZN-PT crystal, ${ }^{7,8}$ consistent with theoretical predictions. ${ }^{9,10}$ Motivated by these studies, it is natural to ask whether the [001] fiber texture is optimal for PMN-PT and PZN-PT ceramics, as far as electromechanical coupling is concerned. We intend to answer this question from a theoretical point of view in this letter.

Compared with their single-crystalline counterpart, polycrystalline ferroelectric ceramics have much more complicated microstructure. They consist of numerous grains, with each grain having its own domain pattern. As such, microstructural phenomena at both grain and domain levels have

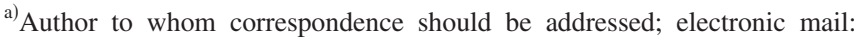
jli2@unl.edu

${ }^{b)}$ Also associated with: Key Laboratory of Advanced Materials \& Rheological Properties of Ministry of Education, Faculty of Materials \& Optoelectronic Physics, Xiangtan University, China.
}

to be accounted for in order to model the ferroelectric ceramic accurately, and there are recent theoretical developments in the modeling of ferroelectric single crystals and ceramics that make this possible. In particular, an energy minimization approach has been applied to construct the engineered domain configuration in a ferroelectric single crystal poled along a nonpolar axis using multirank lamination, ${ }^{9-11}$ allowing the calculation of the electromechanical moduli of a single-crystalline ferroelectrics using lamination theory. Combining this energy minimizationbased lamination theory with an effective medium type selfconsistent model that accounts for the intergranular interactions in ferroelectric ceramics, ${ }^{12,13}$ it is possible to develop a two-scale micromechanical model to calculate the effective electromechanical moduli of textured polycrystalline ferroelectric ceramics, which we report here.

First, let us consider a ferroelectric grain poled along a nonpolar axis by an electric field $\mathbf{E}_{0}$, which favors those variants that minimize $-\mathbf{E}_{0} \cdot \mathbf{p}^{(i)}$, where $\mathbf{p}^{(i)}$ and $\mathbf{e}^{(i)}$ are spontaneous polarization and transformation strain of ferroelectric variants. When those variants are pairwise compatible, laminated domain configurations will be resulted in the grains to minimize their potential energy. ${ }^{10}$ The effective electromechanical moduli of a grain having such a laminated domain configuration is given by ${ }^{10,11}$

$$
\begin{aligned}
& \overline{\mathbf{L}}=\left\langle\mathbf{L}^{-1}\right\rangle^{-1}, \\
& \overline{\mathbf{M}}=\left\langle\mathbf{L}^{-1}\right\rangle^{-1}\left\langle\mathbf{L}^{-1} \mathbf{M}\right\rangle, \\
& \overline{\mathbf{N}}=\left\langle\mathbf{M}^{t} \mathbf{L}^{-1}\right\rangle\left\langle\mathbf{L}^{-1}\right\rangle^{-1}\left\langle\mathbf{L}^{-1} \mathbf{M}\right\rangle+\langle\mathbf{N}\rangle-\left\langle\mathbf{M}^{t} \mathbf{L}^{-1} \mathbf{M}\right\rangle,
\end{aligned}
$$

with 


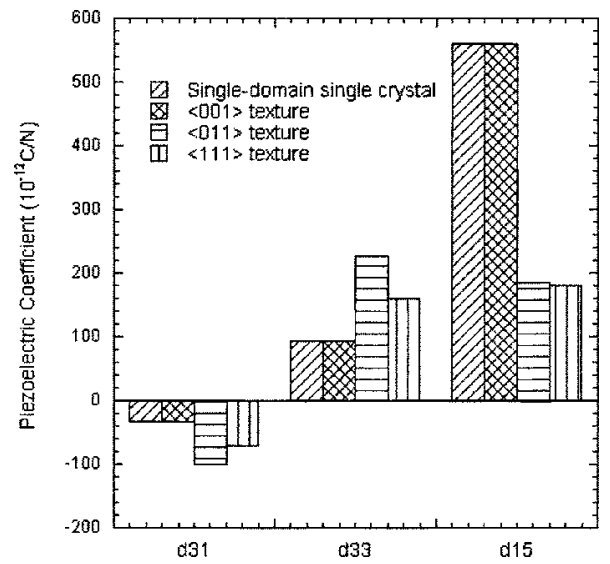

FIG. 1. The piezoelectric coefficients of barium titanate ceramics with fiber textures.

$$
\begin{aligned}
\mathbf{L} & =\left[\begin{array}{llll}
S_{11} & S_{12} & S_{16} & d_{31} \\
S_{12} & S_{22} & S_{26} & d_{32} \\
S_{16} & S_{26} & S_{66} & d_{36} \\
d_{31} & d_{32} & d_{36} & \kappa_{33}^{\sigma}
\end{array}\right], \quad \mathbf{M}^{t}=\left[\begin{array}{llll}
S_{13} & S_{23} & S_{36} & d_{33} \\
S_{14} & S_{24} & S_{46} & d_{34} \\
S_{15} & S_{25} & S_{56} & d_{35} \\
d_{11} & d_{12} & d_{16} & \kappa_{13}^{\sigma} \\
d_{21} & d_{22} & d_{26} & \kappa_{23}^{\sigma}
\end{array}\right], \\
\mathbf{N} & =\left[\begin{array}{lllll}
S_{33} & S_{34} & S_{35} & d_{13} & d_{23} \\
S_{34} & S_{44} & S_{45} & d_{14} & d_{24} \\
S_{35} & S_{45} & S_{55} & d_{15} & d_{25} \\
d_{13} & d_{14} & d_{15} & \kappa_{11}^{\sigma} & \kappa_{12}^{\sigma} \\
d_{23} & d_{24} & d_{25} & \kappa_{12}^{\sigma} & \kappa_{22}^{\sigma}
\end{array}\right] .
\end{aligned}
$$

where $S_{I J}, d_{i J}$, and $\kappa_{i j}^{\sigma}$ are, respectively, elastic compliance, piezoelectric (strain), and dielectric constants (measured at constant stress) of single-domain single crystalline ferroelectric that need to be provided. In the equations, $\langle\cdot\rangle$ is used to denote the volume averaging, and superscript $t$ and -1 are used to denote matrix transpose and inverse, respectively.

The effective electromechanical moduli of each grain can then be used to calculate the effective electromechanical moduli of ferroelectric ceramics using self-consistent model, which leads to ${ }^{12,13}$

$$
\mathbf{G}^{*}=\left\langle\overline{\mathbf{G}}(\theta, \psi, \phi) \mathbf{A}^{\mathrm{dil}}(\theta, \psi, \phi)\right\rangle\left\langle\mathbf{A}^{\mathrm{dil}}(\theta, \psi, \phi)\right\rangle^{-1},
$$

with

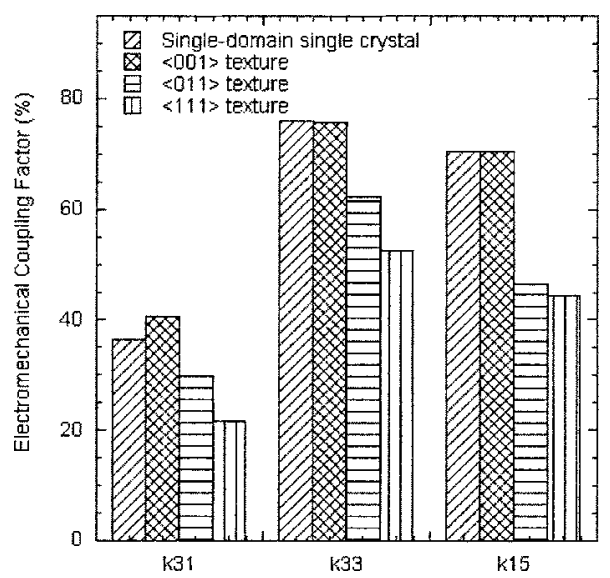

FIG. 2. The electromechanical coupling factors of barium titanate ceramics with fiber textures.
TABLE I. Electromechanical moduli of single-domain single-crystalline barium titanate, ${ }^{a} S: 10^{-12} \mathrm{~m}^{2} / \mathrm{N} ; d: 10^{-12} \mathrm{C} / \mathrm{N} ; \kappa: \kappa_{0}$.

\begin{tabular}{ccccccccccc}
\hline \hline$S_{11}$ & $S_{12}$ & $S_{13}$ & $S_{33}$ & $S_{44}$ & $S_{66}$ & $d_{31}$ & $d_{33}$ & $d_{15}$ & $\kappa_{11}$ & $\kappa_{33}$ \\
\hline 7.38 & -1.39 & -4.41 & 13.1 & 16.4 & 7.46 & -33.72 & 93.95 & 560.7 & 4366 & 132 \\
\hline
\end{tabular}

${ }^{\mathrm{a}}$ See Ref. 17.

$$
\overline{\mathbf{G}}=\left[\begin{array}{cc}
\overline{\mathbf{C}} & \overline{\mathbf{e}} \\
\overline{\mathbf{e}}^{t} & -\overline{\boldsymbol{\kappa}}^{\varepsilon}
\end{array}\right],
$$

where $\overline{\mathbf{C}}, \overline{\mathbf{e}}$, and $\overline{\boldsymbol{\kappa}}^{\varepsilon}$ are, respectively, the effective elastic stiffness, piezoelectric (stress), and dielectric constants (measured at constant strain) of ferroelectric single crystal with laminated domain configuration, which can be converted from $\bar{S}_{I J}, \bar{d}_{i J}$, and $\bar{\kappa}_{i j}^{\sigma}$ calculated from Eq. (1). Due to the anisotropy of crystal, grains at different orientations will have different electromechanical moduli in a global coordinate system, and such dependence on orientation is denoted by $\overline{\mathbf{G}}(\theta, \psi, \phi)$, where $(\theta, \psi, \phi)$ are Euler angles that describe grain's orientation in the global coordinate. In Eq. (2), the dilute concentration factor is given by

$$
\mathbf{A}^{\mathrm{dil}}(\theta, \psi, \phi)=\left[\mathbf{I}+\mathbf{S}(\theta, \psi, \phi) \mathbf{G}^{*-1}\left(\overline{\mathbf{G}}(\theta, \psi, \phi)-\mathbf{G}^{*}\right)\right]^{-1},
$$

where $\mathbf{I}$ is a $9 \times 9$ unit matrix, and $\mathbf{S}(\theta, \psi, \phi)$ is the piezoelectric Eshleby tensor for grain at orientation $(\theta, \psi, \phi)$, which is a function of yet unknown effective electromechanical moduli of the ceramic, $\mathbf{G}^{*}$, and shape and orientation of grain. ${ }^{14,15}$ Equations (2) and (3) are thus coupled together, and need to be solved numerically by iteration in general. For the evaluation of $\mathbf{G}^{*}$, it is noted that the macroscopic properties of ferroelectric ceramics are determined from orientational averaging using orientation distribution function ${ }^{16}$ (ODF) $W(\theta, \psi, \phi)$, which gives the probability of locating a grain at orientation $(\theta, \psi, \phi)$. For example, to average a physical quantity $H(\theta, \psi, \phi)$, we have

$$
\begin{aligned}
\langle H(\theta, \psi, \phi)\rangle= & \int_{0}^{2 \pi} \int_{0}^{2 \pi} \int_{-1}^{1} H(\theta, \psi, \phi) \\
& \times W(\theta, \psi, \phi) d z d \psi d \phi, \quad \text { where } z=\cos \theta,
\end{aligned}
$$

which can be integrated numerically using Gaussian quadrature method. ${ }^{13}$ For the fiber texture we are considering here,

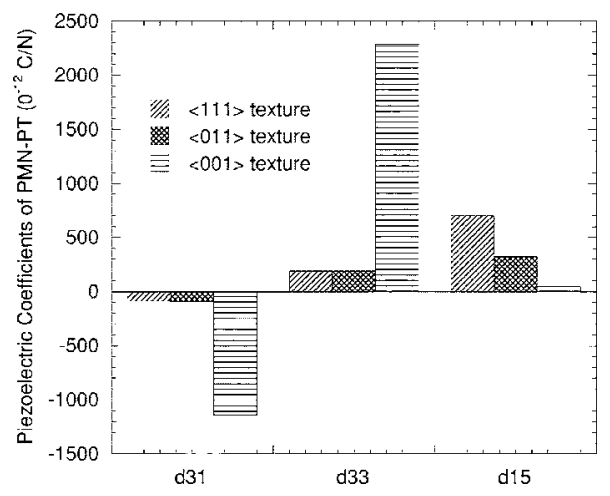

FIG. 3. The piezoelectric coefficients of PMN-PT ceramics with fiber textures. 
TABLE II. Electromechanical moduli of single-domain single-crystalline PMN-PT, ${ }^{\mathrm{a}} S: 10^{-12} \mathrm{~m}^{2} / \mathrm{N} ; d$ : $10^{-12} \mathrm{C} / \mathrm{N} ; \kappa: \kappa_{0}$.

\begin{tabular}{ccccccccccccc}
\hline \hline$S_{11}$ & $S_{12}$ & $S_{13}$ & $S_{14}$ & $S_{33}$ & $S_{44}$ & $S_{66}$ & $d_{31}$ & $d_{33}$ & $d_{15}$ & $d_{22}$ & $\kappa_{11}$ & $\kappa_{33}$ \\
\hline 62.16 & -53.85 & -5.58 & -166.24 & 13.34 & 510.98 & 232.02 & -90 & 190 & 4100 & 1340 & 3950 & 640 \\
\hline \hline
\end{tabular}

${ }^{\mathrm{a}}$ See Ref. 19.

$$
W(\theta, \psi, \phi)=\delta(\theta),
$$

where $\delta(\theta)$ is delta function, which suggests that all grains are aligned along a particular crystallographic direction as the $x_{3}$ axis of the ceramic, and are randomly oriented in the $x_{1}-x_{2}$ plane. The resulting ceramic thus possesses transversely isotropic symmetry.

We applied this two-scheme micromechanical model to calculate the effective electromechanical moduli of piezoelectric ceramics $\mathrm{BaTiO}_{3}$ and PMN-PT with various fiber textures, including [001], [011], and [111], where each grain is free to rotate around the $x_{3}$ axis. For barium titanate, the single-domain single-crystalline data ${ }^{17}$ are listed in Table I, which was used as input to calculate the effective piezoelectric coefficients of barium titanate ceramics with fiber textures, shown in Fig. 1. It is interesting to note that the ceramic with either [011] or [111] fiber texture has much higher piezoelectric coefficients $d_{31}$ and $d_{33}$ than ceramics with [001] fiber texture, and ceramics with [011] fiber texture have the highest $d_{31}$ and $d_{33}$. This is consistent with observation in single-crystalline barium titanate poled along either the [011] or [111] axis, which has higher $d_{31}$ and $d_{33}$ than single-domain single crystals poled along the [001] axis. On the other hand, the electromechanical factors $k_{31}$ $=d_{31} / \sqrt{\kappa_{33}^{\sigma} S_{11}}, k_{33}=d_{33} / \sqrt{\kappa_{33}^{\sigma} S_{33}}$, and $k_{15}=d_{15} / \sqrt{\kappa_{11}^{\sigma} S_{44}}$ demonstrate different trend, as shown in Fig. 2, with [001] fiber texture resulting in highest coupling factor, and [111] fiber texture resulting lowest coupling factor. It is also interesting to note that the [001]-textured ceramic has an even higher electromechanical coupling factor than that of single-domain single crystal, resulting from the intergranular electrome-

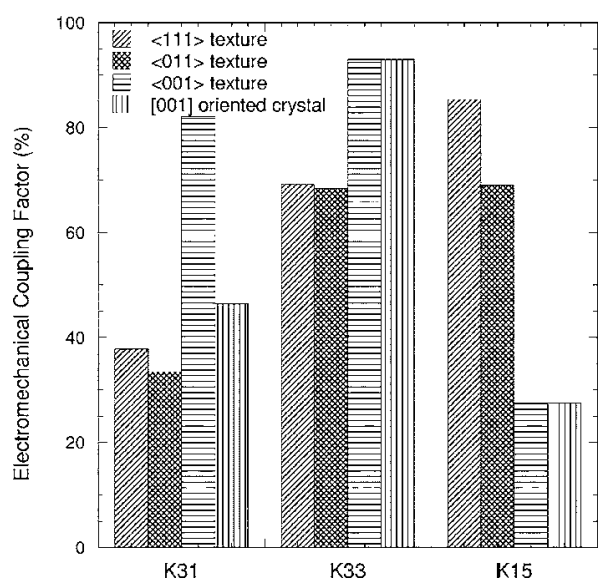

FIG. 4. The electromechanical coupling factors of PMN-PT ceramics with fiber textures. chanical interactions that lead to stiffening in the $x_{1}-x_{2}$ plane. This is consistent with theoretical analysis ${ }^{18}$ that [001]textured tetragonal ceramic has identical $d_{31}, d_{33}, S_{33}$, and $\kappa_{33}$ as single crystal, yet different $S_{11}$ and $S_{12}$.

For PMN-PT ceramics, we used the single-domain single-crystalline data ${ }^{19}$ listed in Table II as input to calculate their effective piezoelectric coefficients, shown in Fig. 3. It is interesting to notice that although a higher piezoelectric coefficient $d_{32}$ and electromechanical coupling factor $k_{32}$ were reported in [011]-oriented PMN-PT crystal, ${ }^{7-10}$ the [011]textured PMN-PT ceramics show much inferior piezoelectric coefficients $d_{31}$ and $d_{32}$. This is because for a [011]-oriented PMN-PT single crystal, $d_{31}$ and $d_{32}$ have opposite signs, ${ }^{10}$ and thus they tend to cancel each other out when these single-crystalline grains are assembled into a ceramic, leading to much smaller piezoelectric strain and piezoelectric coefficients in the [011]-textured ceramics. On the other hand, it is observed that the electromechanical coupling factor $k_{31}$ of [001]-textured ceramic is much higher than that of [001]oriented single crystal, as shown in Fig. 4, again due to the stiffening of the ceramic in the $x_{1}-x_{2}$ plane.

The work is partially supported by U.S. National Science Foundation and NASA Nebraska Space Grant and EPSCoR seed grant. We also acknowledge Trans-Century Training Program Foundation for Talents (No:[2002]48), China and Furong Scholars Programme of Hunan Province, China.

${ }^{1}$ S. E. Park and T. R. Shrout, J. Appl. Phys. 82, 1804 (1997).

${ }^{2}$ J. Kuwata, K. Uchino, and S. Nomura, Jpn. J. Appl. Phys., Part 1 21, 1298 (1982).

${ }^{3}$ E. M. Sabolsky, A. R. James, S. Kwon, S. Trolier-McKinstry, and G. L. Messing, Appl. Phys. Lett. 78, 2551 (2001).

${ }^{4}$ E. M. Sabolsky, S. Trolier-McKinstry, and G. L. Messing, J. Appl. Phys. 93, 4072 (2003).

${ }^{5}$ S. W. Sun, X. M. Pan, P. C. Wang, L. H. Zhu, Q. W. Huang, D. L. Li, and Z. W. Yin, Appl. Phys. Lett. 84, 574 (2004).

${ }^{6}$ G. L. Messing, S. Trolier-McKinstry, E. M. Sabolsky, C. Duran, and S. Kwon, Science 29, 45 (2004).

${ }^{7}$ T. Liu and C. Lynch, Acta Mater. 51, 407 (2003).

${ }^{8}$ R. Zhang, B. Jiang, and W. Cao, J. Phys. Chem. Solids 65, 1083 (2004).

${ }^{9}$ D. Liu and J. Y. Li, Appl. Phys. Lett. 84, 3930 (2004).

${ }^{10}$ J. Y. Li and D. Liu, J. Mech. Phys. Solids 52, 1719 (2004).

${ }^{11}$ D. Liu and J. Li, Appl. Phys. Lett. 83, 1193 (2003).

${ }^{12}$ M. L. Dunn, J. Appl. Phys. 78, 1533 (1995).

${ }^{13}$ J. Y. Li, J. Mech. Phys. Solids 48, 529 (2000).

${ }^{14}$ M. L. Dunn and M. Taya, Proc. R. Soc. London, Ser. A 443, 265 (1993).

${ }^{15}$ J. D. Eshelby, Proc. R. Soc. London, Ser. A 241, 376 (1957).

${ }^{16}$ R. J. Roe, J. Appl. Phys. 36, 2024 (1965).

${ }^{17}$ M. Zgonik, P. Bernasconi, M. Duelli, R. Schlesser, P. Gunter, M. H. Garrett, D. Rytz, Y. Zhu, and X. Wu, Phys. Rev. B 50, 5941 (1994).

${ }^{18}$ J. Y. Li, M. L. Dunn, and H. Ledbetter, J. Appl. Phys. 86, 4626 (1999).

${ }^{19}$ R. Zhang, B. Jiang, and W. Cao, Appl. Phys. Lett. 82, 787 (2003). 